\title{
Association of ovarian stimulation and embryonic aneuploidy in in vitro fertilization cycles with preimplantation genetic testing: A narrative systematic review
}

\author{
Jorge Rodriguez-Purata ${ }^{1,2}$, Maria Jose Gomez-Cuesta ${ }^{1}$, Enrique Cervantes-Bravo ${ }^{1,2}$ \\ ${ }^{1}$ Clinica de la Fertilidad "CdelaF", Prolongacion Vasco de Quiroga 4001, Torre A Piso 10, Colonia Santa Fe, Cua- \\ jimalpa de Morelos, 05370, Mexico City, Mexico \\ ${ }^{2}$ Centro Medico ABC, Avenida Carlos Graef Fernández 154, Colonia Santa Fe, Cuajimalpa de Morelos, 05300, \\ Mexico City, Mexico
}

\begin{abstract}
The impact of gonadotropins used for COS on the rate of embryo aneuploidy in patients without the negative effects of age as a confounding factor, is still a subject of lively debate. We ran a systematic search for studies in MEDLINE, PubMed, Google Scholar and the Cochrane Library. A librarian coordinated the search in December of 2020. We included all original peer-reviewed papers in English, irrespective of study-design. There were no restrictions concerning method of amplification or platform used to analyze the amplified DNA. We used the PICO model to select the study population. We included women/ couples submitted to COS for IVF with the intention to genetically analyze her/their embryos through PGT. The primary outcome was the rate of aneuploidy. We used the Newcastle-Ottawa scale (NOS) score to evaluate the quality of the studies included. The search yielded 73 citations, and 14 were eligible for analysis, which included data on 4805 cycles. Media quality NOS score was 8. Although it has been demonstrated that natural cycles are associated with aneuploidy, it does seem that more robust stimulations are indeed associated with a higher proportion of aneuploidy. Nevertheless, a higher response is associated with an increased number of euploid embryos available for transfer, which translates into more embryotransfer cycles with a prospective higher cumulative live birth rate. Further evidence is needed to ascertain if there is a negative impact of COS, especially at the cellular level.
\end{abstract}

Keywords: aneuploidy, in vitro fertilization, assisted reproductive techniques, ovarian stimulation, preimplantation diagnosis, preimplantation genetic testing

\section{INTRODUCTION}

Since the pioneering days of in vitro fertilization (IVF) (Trounson et al., 1981), controlled ovarian stimulation (COS) has been an integral part of assisted reproductive technology (ART) treatment. Primarily, the goal of COS is to induce ongoing development of multiple dominant follicles in a single cycle and to mature as many oocytes possible to improve chances for conception either in vivo (empirical ovarian stimulation with or without intrauterine insemination) or in vitro (with IVF) (Macklon et al., 2006). Although several advantages have been thoroughly described (Macklon et al., 2006; Drakopoulos et al., 2016; Rodriguez-Purata et al., 2016), data is still missing regarding the association of COS and the incidence of embryonic aneuploidy.

Commensurate with the developments in COS (MackIon et al., 2006), the more recent implementation of preimplantation genetic testing (PGT) of embryos introduced by Handyside et al. (1989) into the available strategies for treating infertile patients, is allowing the checking of all 24 chromosomes within an embryo before transfer. To date, several improvements have been made to the technology (Scott et al., 2013; Sermon et al., 2016) that have enhanced its validity and propelled its adoption (De Rycke et al., 2015). But even with these improvements, its true value still remains unknown (Practice Committees of the American Society for Reproductive Medicine and the Society for Assisted Reproductive Technology, 2018; Munné et al., 2019).

As a result of the improvements made to the technology, more information is recently available regarding the impact of COS on embryonic aneuploidy. This systematic review assesses the current evidence presented on critical clinical questions concerning the association of COS in women undergoing IVF coupled with PGT and embryonic aneuploidy.

Origin and Mechanisms of Embryonic Aneuploidy in Humans

One of the main concerns is that exogenous follicle stimulating hormone (FSH) may be correlated with an increased risk of embryonic aneuploidy (Elbling \& Colot, 1985; Sato \& Marrs, 1986; Edgar et al., 1987). Whereas animal studies seem to support an association (Santaló et al., 1986), human studies have been more conflicting (Baart et al., 2007; Verpoest et al., 2008; Rubio et al., 2010; Kyrou et al., 2011; Massie et al., 2011; Ata et al., 2012; Labarta et al., 2012; 2017; Barash et al., 2017; Sekhon et al., 2017; Venetis et al., 2019).

Almost all recent information on the chromosomal status of the early human embryo has been derived from the outcome of PGT and follow-up studies thereof. Overall, ART has helped us learn so much about aneuploidy (Nagaoka et al., 2012; Rodriguez-Purata et al., 2015), but it makes us think it is even more prevalent. Before ART, knowledge came essentially from newborns, stillbirths, and abortions (Nagaoka et al., 2012). Nowadays, ART provides data of the stage at which segregation errors become evident, from female meiosis to the blastocyst stage of preimplantation embryo development (Capalbo et al., 2013).

Due to the prevalence and significance of aneuploidy in humans, it is important to understand the origins and mechanisms of development. For a more in-depth analysis, please refer to (Delhanty, 2005; Fragouli et al., 2013; Taylor et al., 2014).

\section{Possible Mechanisms for Aneuploidy by cOS}

The effect of high doses of gonadotropins is not yet well-understood, especially at the most basic level of organization - the cellular level. In theory, it has been speculated that high doses of exogenous gonadotropins administered over a prolonged interval interfere with natural selection, generating $\mathrm{FSH}$-induced chromosome 
dysfunction in oocytes or recruitment of poor-quality oocytes when the natural selection process of a dominant follicle is superseded by COS (Nagaoka et al., 2012).

\section{Evidence from non-human models}

COS influence has been well-characterized in animals, mainly in rodents, and has shown that intense COS could lead to poorer embryonic development potential, and could increase the rate of chromosomal defects. Mice exposed to high doses of gonadotropins had reduced blastulation, a higher rate of chromosomal aberrations, and increased embryonic degeneration (Vogel \& Spielmann, 1992). In contrast, other animal studies have shown a lack of difference in the incidence of nondisjunction in mice oocytes obtained after COS versus natural cycles (Golbus, 1981). More recently, experimental evidence in mice showed that alterations in the multiple signals that regulate folliculogenesis could change the later stages of oocyte development, increasing the risk of poor chromosomal segregation in subsequent meiotic divisions (Hodges et al., 2002).

\section{Evidence from human studies}

Unfortunately, human studies to date bear some degree of conflicting results and conflicting conclusions, possibly due to significant differences in study design and the technology used to assess ploidy. In general, COS has been proposed to influence oocyte maturation and completion of meiosis, potentially mediating chromosomal aneuploidy and mosaicism (Verberg et al., 2009). Additionally, elevated serum estradiol levels and oocyte yield have been associated with increased blastomere multinucleation (Jackson et al., 1998).

Recent observations suggest that inaccuracies in the chromosomal segregation mechanism in oocytes are often involved, and this process is triggered by maternal age (Hassold \& Hunt, 2001; Champion \& Hawley, 2002). Inaccuracies in chromosome segregation in oocytes may be responsible for embryonic aneuploidy, and may be induced by failures in the mechanisms that control cell cycle, thus affecting the entire chromosome segregation process (Barlow \& Hultén, 1998; Tease et al., 2002); whereas segregation of particularly unstable bivalents responsible for aneuploidies arising during the first meiotic division can be caused by synaptic errors between specific homologous chromosomes, or a reduction in the number of chiasms due to a low recombination rate (Nicolaidis \& Petersen, 1998; Hassold \& Hunt, 2001; Champion \& Hawley, 2002; Obradors et al., 2010). The COS used for IVF could be responsible for these abnormalities in chromosome segregation; hence the presence of aneuploidy and mosaicism in embryos due to changes to the cytoskeleton and/or the mitotic spindle in the first embryonic division of bipronuclear zygotes (Munne et al., 1997).

Furthermore, analysis of human gametes revealed that embryo aneuploidy is caused primarily by an error-prone meiotic chromosomal segregation mechanism in oocytes. If COS can exert any deleterious effect on the embryo, this may be because it induces meiotic errors in the oocyte (a uniformly aneuploid monosomal, trisomic, haploid, or polyploid embryo), but not mitotic errors after fertilization (mosaicism) (Frumkin et al., 2008). At the genomic level, the increased incidence of genomic imprinting disorders in oocytes after ovulation induction and in vitro maturation may be due not only to maternal factors such as age, but also to external factors such as the biochemistry of the abnormal or suboptimal follicular fluid and cytoplasmic development (Sato et al., 2007).

\section{MATERIALS AND METHODS}

\section{Protocol and Registration}

The study complied with the Preferred Reporting Items for Systematic Reviews and Meta-Analyses (PRISMA) guidelines (Moher et al., 2009). The study protocol was registered at http://www.crd.york.ac.uk/PROSPERO/ (CRD42019120803) before starting the review. This study was exempted from the institutional review board approval, because it is a systematic review.

\section{Eligibility Criteria}

We used the PICO (Patients, Intervention, Comparison and Outcomes) model to select the study population (Schardt et al., 2007), and we included women/couples who underwent COS for an IVF cycle with the intention to genetically analyze her/their embryos through PGT.

\section{Search Strategy}

The authors performed a systematic search of the literature for studies appropriate to the clinical question on the Medical Literature Analysis and Retrieval System Online (MEDLINE), ClinicalTrials.gov, PubMed, Google Scholar, and the Cochrane Library (http://www.cochranelibrary. com). We included a combination of MeSH terms and/or text words relevant to the association of COS with embryonic aneuploidy in the setting of an IVF cycle with PGT in the search builder (Supplemental Table 1). The authors additionally checked citations on the Web of Science and manually searched the references of the papers. Searches were coordinated by an expert librarian and a statistician in September'20. Search updates were conducted in December'20. All records were screened for eligibility by two independent reviewers. We included all original English, peer-reviewed articles, irrespective of study-design, were included.

\section{Study Selection}

Two reviewers (J.R-P./E.C-B) independently evaluated titles and abstracts. Duplications were removed using the Zotero online software and manually, when needed. Final inclusion/exclusion decisions were made upon examining the papers in full. We resolved discrepancies by discussion and consensus between authors, with the involvement of another author (MJ.G-C). To acquire the highest level of evidence, we selected randomized trials and observational studies, including cohort- and case-control studies. Trials published only as abstracts, letter to the editor, editorials, or studies retracted from the literature after their publication were excluded upfront. Systematic reviews were also excluded, but we checked the references first.

Intervention studies were eligible if: 1) randomized controlled trials (RCTs), non-RCTs, prospective observational studies, or cohort studies; 2) assessed the impact of COS on generating aneuploid embryos and/or conceptions; 3) under any type of stimulation protocol (long agonist, antagonist, microflare, estrogen priming, progesterone-primed, etc.) comparing the study group vs. no COS or COS with a reduced dose; and 4) collected the status of the biopsied embryos as euploid/aneuploid endpoint. There were no restrictions in terms of amplification method, or the platform used to analyze the amplified DNA.

\section{DATA Extraction and Quality Assessment}

Eligible records were systematically collected and imported into an electronic database by two reviewers (J.R-P. and E.C-B), using predefined data fields and collected in a standardized data extraction form. This included the following data: year of publication, study design, study period, intervention, number of patients (total, exposed and 
unexposed), PGT platform, biopsy day (cleavage or blastocyst stage biopsy), number of cells biopsied, age criteria, general inclusion criteria, control and study groups, average of oocytes retrieved, total number of euploid embryos, euploid/aneuploid ratio. Additionally, for intervention studies, allocation concealment and blinding were also recorded. In case of multiple publications we collected, the most up-to-date or comprehensive information.

The studies included were further subclassified according to the stimulation protocol: COS vs. no COS; low vs. conventional dose; COS under a long-agonist vs. antagonist; FSH-only vs. luteinizing hormone (LH)-containing gonadotropins; trigger medication used; and according to ovarian response. Within subgroups, special attention was given to oocytes retrieved, total number and average of euploid embryos, and proportion of euploid/aneuploid embryos.

When specifically comparing COS vs. no COS and conventional vs. reduced dose, we ran an intention-to-treat (ITT) analysis (Sedgwick, 2015) in order to provide a comparable analysis between groups and specifically answer the following question: if a patient decides to undergo a PGT cycle, which strategy provides the higher odds of developing a euploid embryo? ITT analysis means all patients who were enrolled and randomly allocated to treatment were included in the analysis and were analyzed in the groups to which they were initially randomized. This analysis is particularly relevant in this review, given that it has been previously established that patients who do not reach retrieval or biopsy stage are potentially aneuploid oocytes/ embryos (Qi et al., 2014).

\section{Appraisal of Certainty of Evidence}

Two authors (J.R-P. /E.C-B.) independently assessed the risk bias of each study. We used the Newcastle-Ottawa scale (NOS) to evaluate the case-control or cohort studies included, and judgment on each one was passed according to three issues: selection of the study group, comparability between groups and the ascertainment of outcomes and exposures of interest (Hartling et al., 2012). This scale gives up to 9 stars to each study and classifies them as low quality (0-4 stars), moderate quality (5-6 stars) and high quality (7-9 stars) (Wells et al., 2014) (Supplemental Table 2).

The evaluation was performed independently by two reviewers (J.R-P., E.C-B), while any disagreement was resolved by discussion between the two parties or by including a third reviewer (MJ.G-C.).

We did not run a meta-analysis because of the large heterogeneity in the definitions of COS exposure, biopsy stage, and platform used.

\section{RESULTS}

\section{Study Selection}

The literature search retrieved 74 citations, of which 11 were eligible for further consideration after screening titles and abstracts. Cross-reference checking resulted in an additional inclusion of four papers. Haaf et al. (2009) was not detected because it does not include any of the Medical Subject Headings (MeSH) terms or text words related to IVF. Rubio et al. (2010) and Thorne et al. (2019) were not detected because they did not include any of the $\mathrm{MeSH}$ terms or text words related to the PGT procedure, type of analysis or type of platform. Ata et al. (2012) was not detected because it does not include any of the MeSH terms or text words related to COS (Figure 1).

\section{Description of Studies Included}

Overall, 15 studies published before December 2019 fulfilled our inclusion criteria, and included data on 5344 cycles (Baart et al., 2007; Verpoest et al., 2008; Weghofer et al., 2008; 2009; Haaf et al., 2009; Rubio et al., 2010; Kyrou et al., 2011; Massie et al., 2011; Ata et al., 2012; Labarta et al., 2012; 2017; Barash et al., 2017; Sekhon et al., 2017; Thorne et al., 2019; Venetis et al., 2019) were included (Table 1). There are five studies from the USA, three from Spain, two from Belgium and Austria/USA, respectively, and one from Australia, Germany and the Netherlands, respectively. There is one RCT (Baart et al., 2007), four prospective non-randomized trials (Verpoest et al., 2008; Rubio et al., 2010; Labarta et al., 2012; 2017) and ten retrospective studies (Weghofer et al., 2008; 2009; Haaf et al., 2009; Kyrou et al., 2011; Massie et al., 2011; Ata et al., 2012; Barash et al., 2017; Sekhon et al., 2017; Thorne et al., 2019; Venetis et al., 2019). In terms of platform used, three studies used array comparative genomic hybridization (aCGH) (Ata et al., 2012; Thorne et al., 2019; Venetis et al., 2019), one study used quantitative polymerase chain reaction-based comprehensive chromosome screening (qPCR-based CCS) (Sekhon et al., 2017), one study used single nucleotide polymorphism (SNPs) (Barash et al., 2017), and ten underwent fluorescence in situ hybridization (FISH) analysis. In terms of biopsy stage, three studies included patients whose embryos underwent biopsy at the blastocyst stage (Barash et al., 2017; Sekhon et al., 2017; Thorne et al., 2019), nine studies included patients whose embryos underwent biopsy at the cleavage stage, and one study included both stages, although analyzed independently (Ata et al., 2012). Lastly, two studies providing an indirect measurement of embryonic aneuploidy in relation to COS exposure: one study analyzed polar bodies (Haaf et al., 2009) and one study analyzed products of conception (POC) (Massie et al., 2011).

\section{Principal Findings}

\section{Non-stimulated vs. Stimulated IVF}

Three studies compared the incidence of aneuploidy in non-stimulated $v s$. stimulated cycles. First, Verpoest et al. (2008) prospectively followed 30 patients who underwent a natural cycle, of which 20 reached retrieval, 19 oocytes were successfully retrieved (0.6/patient), 15 MIIs fertilized and developed into 11 embryos which can biopsied: 4 aneuploid, 6 euploid (0.2/patient) and 1 undiagnosed. There was no control group. Aneuploidy rate, performed by FISH analysis, was $36.4 \%$ (Verpoest et al., 2008). An ITT analysis yielded $20 \%(6 / 30)$ of the patients with an euploid embryo.

Similarly, Massie et al. (2011) analyzed the POC of missed abortions from infertility patients who conceived naturally $(n=50)$ versus infertility patients who conceived under FSH stimulation $(n=179)$. Despite similar age in both groups, the authors reported aneuploidy in POCs in $70 \%$ of the samples when patients conceived naturally, vs. $63 \%$ after FSH stimulation for IVF (Massie et al., 2011). Although this study provides an indirect comparison, it shows that patients without stimulation are at similar risk of generating abnormal embryos.

Lastly, Labarta et al. (2012) performed a prospective cross-over study in which the same donor underwent an unstimulated and a stimulated COS cycle. The aneuploidy rate reported was $35.3 \%(18 / 51)$ in the unstimulated group and $40.6 \%(123 / 303)$ in the stimulated group. In terms of euploid embryos per patient, there was 1.8 in the unstimulated group vs. 3.9 in the stimulated group. Of note, the stimulated group included 47 patients and $97.9 \%$ reached the biopsy stage. Conversely, in the unstimulated group, 185 donors were enrolled and only $27.6 \% \quad(n=51)$ reaching biopsy $[65.4 \%(n=121)$ reached retrieval, $53.5 \%$ $(n=99)$ had an oocyte retrieved; $48.1 \%(n=89)$ had an MII retrieved; $30.8 \%(n=57)$ fertilized] (Labarta et al., 2012). Considering that almost all arrested embryos are 
not euploid (Qi et al., 2014), the proportion of included patients who achieved an euploid embryo was actually $58.6 \%$ $(180 / 307)$ in the stimulated group vs. $16.2 \%(30 / 185)$ in the unstimulated group. Therefore, the actual aneuploidy rate in the unstimulated cycle was $76.8 \%$ (76/99 oocyte retrievals) vs. $40.6 \%(123 / 303)$ in the stimulated cycle. Correspondingly, the true number of euploid embryos per patient of the unstimulated was 1.0 vs. 3.9 in the stimulated group.

In summary, the absence of stimulation does not preclude embryonic aneuploidy. Special attention should be given when comparing patients, because most of the times patients that do not reach biopsy stage are not taken into account in the final analysis.

\section{Low vs. Conventional Gonadotropin Dose}

Two studies have compared a conventional vs. a reduced dose. The first study was published by Baart et al. (2007), and it is the only RCT included in this systematic review. The authors compared 111 patients randomized to a conventional $(n=44)$ versus reduced $(n=67)$ protocol with PGT under FISH analysis. Interestingly, the authors terminated the study prematurely due to a significantly lower embryo aneuploidy rate per patient observed after a reduced dose (Baart et al., 2007). There are two key comments to this manuscript: first, the authors reported that conventional stimulation was associated with a $38 \%$ euploidy rate $(61 / 159)$, versus $50 \%$ euploidy rate $(71 / 143)$ with the reduced dose $(p=0.02)$. The authors stated that the reason for interruption was that we met a Pocock critical bound (the $p$-value was <0.0354). Of note, the $p$-value for that comparison is 0.048 . Also, when analyzing the tables included, the aneuploidy rate was actually $26 \%(41 / 159)$ with conventional stimulation, versus $22 \%$ (31/143) with a reduced dose, with a $p$-value of 0.4 . Second and most importantly, if the authors would have done an ITT analysis, the results would have been more valid and practical for advising patients. The authors assert that both groups had 1.8 euploid embryos available for transfer. If all patients would have been considered, patients undergoing conventional stimulation would have had 2.5 euploid embryos and patients undergoing mild stimulation would have had 1.4 euploid embryos (table II of Baart et al.'s manuscript, divided into 44 and 67 patients, respectively, instead of dividing the results in 33 and 40 patients, who reach biopsy stage, respectively) (Table 1 ).

Later, Rubio et al. (2010) published a prospective crossover study including 32 donors who underwent a COS with standard dose (225 IU) and a subsequent cycle with a $30 \%$ dose reduction (150 IU). Twenty-two donors completed both cycles, with a $31.3 \%$ cancellation rate in the reduced-dose group. The authors found significant differences in euploidy rate: $22.5 \%$ (standard dose) vs. $16.1 \%$ (reduced dose). Additionally, the authors reported a statistical difference in the proportion of blastocysts per biopsied embryo (66.8\%) $(139 / 296)$ vs. $75.6 \%(115 / 152), p=0.04)$ in favor of the reduced dose. And in terms of clinical outcomes, the authors reported a pregnancy rate (PR) per transfer of $56.0 \%(14 / 25)$ with a standard dose vs. $52.2 \%$ $(12 / 23)$ with a reduced dose (Rubio et al., 2010). Unfortunately, satisfactory conclusions cannot be drawn because the authors also did not perform an ITT analysis. Considering all patients assigned per arm (included the 10 patients in the reduce dose who were canceled), the proportion of blastocysts per biopsied embryos would have been $69.3 \%$ (205/296) versus $75.6 \%(115 / 152)$, rendering a $p>0.05$. Additionally, if we take into account the biopsied embryos $(n=82)$ from the 10 patients under conventional-dose, there are an additional 33 euploid embryos, which translates into 3.1 euploid embryos available per donor in the standard dose vs. 1.8 in the reduced-dose (instead of 3.1 vs. 2.7). And most importantly, PR per transfer would have been $63.9 \%(23 / 36)$ in the standard-dose group vs. $52.2 \%$ $(12 / 23)$ in the reduced-dose group (Rubio et al., 2010).

Although both studies conclude that a reduced dose is associated to a lower proportion of aneuploidy, it is not clear that, when including all patients in the analysis, a reduced dose would provide a higher euploidy rate. Studies with an ITT design are needed to truly determine a strategy's aneuploidy rate and total number of embryos biopsied.

\section{GnRH Agonist versus GnRH Antagonist}

Only one study compared the incidence of aneuploidy correlated to the type of pituitary suppression. Kyrou et al. (2011) compared 694 consecutive cycles in patients $\leq 37$ years undergoing a long-agonist $(n=320)$ vs. antagonist protocol ( $n=374$ cycles) under the same COS criteria. The Patients' embryos underwent D3 biopsy, FISH analysis and D5 embryo transfer (ET). The aneuploidy rate was statistically similar between the embryos of the two study groups $(49.9 \pm 28.1$ vs. $50.2 \pm 26.6 ; p=0.922)$. A multivariate linear regression analysis confirmed that the total abnormality ratio was not influenced by the type of stimulation when simultaneously adjusting for age, rank of trials, indication for PGT, total gonadotropin amount, number of COCs, and number of 2PN embryos (Kyrou et al., 2011).

Unfortunately, the authors only included patients who reached biopsy; thus, an ITT analysis could not be performed. The authors concluded that there was no difference in the proportion of aneuploidy when using an agonist or antagonist protocol (Kyrou et al., 2011).

\section{FSH-Only versus $\mathbf{L H}$-Containing Gonadotropins}

Two studies have weighed the impact of LH-containing gonadotropins on the chromosomal status of preimplantation embryos during IVF (Weghofer et al., 2008; 2009). First, Weghofer et al. (2008) studied the effect of COS with FSH-only medications $(n=52)$ vs. human menopausal gonadotropin (hMG) $(n=52)$ in patients undergoing a long agonist protocol. Overall, the rate of euploidy, calculated on a per-patient basis, was significantly higher in patients under hMG $(45.3 \%$ versus $69.8 \%$, respectively, $p<0.01)$. Interestingly, the total number of euploid embryos was similar (FSH 3.1 vs. hMG 3.3). This difference is explained because patients who utilized rFSH produced significantly more aneuploid and complex abnormal embryos than patients undergoing hMG stimulation ( $\mathrm{rFSH}$ : aneuploid embryos 2.2, complex abnormal embryos 2.9; hMG aneuploid embryos 0.9 , complex abnormal embryos $1.2, p<0.01$ ). Therefore, the proportion was in favor of the hMG group. A linear multi-regression analysis (age, IVF attempts, diagnosis, type and dosage of gonadotropins used, oocytes retrieved, usable embryos) confirmed that FSH-only stimulation influenced aneuploidy rates $(p<0.02)$ (Weghofer et al., 2008).

The same group evaluated patients undergoing IVF under an antagonist protocol (Weghofer et al., 2009). Both groups produced statistically similar numbers of euploid embryos (FSH, 2.1 vs. FSH/hMG, 1.9) and similar euploidy rates, calculated on a per-patient basis (FSH: 29.4\% vs. FSH/hMG: $25.7 \%$ ). After a linear multi-regression analyses, ploidy status was not impacted by the gonadotropin used (FSH vs. FSH/hMG), the indication for PGD (after correction for age), nor the duration of GnRH antagonist.

Consequently, with these results and given that during an antagonist cycle LH is not suppressed at the beginning of the cycle, we can infer that initial gonadotropin-dependent follicle selection is maintained during antagonist cycles, whereas it might be in agonist cycles, in which endogenous LH concentrations are reduced very in the cycle. 
Table 1. Characteristics of the studies included.

\begin{tabular}{|c|c|c|c|c|c|c|}
\hline Author & Country & Study Period & Design & Intervention & $\begin{array}{l}\text { Control } \\
\text { Group }\end{array}$ & Study Group \\
\hline $\begin{array}{l}\text { Baart } \\
\text { et al., } 2007\end{array}$ & Netherlands & Dec'02-Aug'05 & RCT & $\begin{array}{l}\text { Low vs. Conventional } \\
\text { Stimulation }\end{array}$ & $\begin{array}{l}150 \mathrm{IU} / \mathrm{an}- \\
\text { tagonist }\end{array}$ & $\begin{array}{l}225 \text { IU / long } \\
\text { agonist }\end{array}$ \\
\hline $\begin{array}{l}\text { Weghofer } \\
\text { et al., } 2008\end{array}$ & Austria/USA & Jan'04-Jun'05 & Retrospective & $\begin{array}{c}\text { FSH vs. LH-containing } \\
\text { gonadotropins/agonist protocol }\end{array}$ & FSH & hMG \\
\hline $\begin{array}{l}\text { Verpoest } \\
\text { et al., } 2008\end{array}$ & Belgium & Oct'05-Apr'06 & $\begin{array}{l}\text { Prospective } \\
\text { observational } \\
\text { study }\end{array}$ & $\begin{array}{l}\text { Aneuploidy in Unstimulated } \\
\text { Cycles }\end{array}$ & $\begin{array}{l}\text { No control } \\
\text { group }\end{array}$ & $\begin{array}{l}\text { Unstimulated } \\
\text { cycles }\end{array}$ \\
\hline $\begin{array}{l}\text { Haaf } \\
\text { et al., } 2009\end{array}$ & Germany & Jan'02-Dec'05 & Retrospective & $\begin{array}{c}\text { Oocyte aneuploidy } \\
\text { by age and response } \\
(1-5,6-10, \text { or }>10 \text { oocytes })\end{array}$ & $<35$ years & $\begin{array}{l}35-40 \text { years; } \\
>40 \text { years }\end{array}$ \\
\hline $\begin{array}{l}\text { Weghofer } \\
\text { et al., } 2009\end{array}$ & Austria/USA & Jan'03-Jun'05 & Retrospective & $\begin{array}{c}\text { FSH vs. LH-containing } \\
\text { gonadotropins/antagonist } \\
\text { protocol }\end{array}$ & FSH & hMG \\
\hline $\begin{array}{l}\text { Rubio } \\
\text { et al., } 2010\end{array}$ & Spain & NA & Crossover study & $\begin{array}{c}\text { 1st cycle normal dose vs. 2nd } \\
\text { cycle } 30 \% \text { reduce dose, } 3 \\
\text { months apart }\end{array}$ & $\begin{array}{l}225 \mathrm{IU} / \text { long } \\
\text { agonist }\end{array}$ & $\begin{array}{l}150 \mathrm{UI} / \\
\text { antagonist }\end{array}$ \\
\hline $\begin{array}{l}\text { Kyrou } \\
\text { et al., } 2011\end{array}$ & Belgium & Oct'92-Dic'06 & Retrospective & $\begin{array}{c}\text { GnRH agonist vs. GnRH } \\
\text { Antagonist }\end{array}$ & Agonist & Antagonist \\
\hline $\begin{array}{l}\text { Massie } \\
\text { et al., } 2011\end{array}$ & USA & Jan'99-Aug'07 & Retrospective & $\begin{array}{l}\text { Aneuploidy in miscarriages } \\
\text { from stimulation vs. no } \\
\text { stimulation }\end{array}$ & $\begin{array}{c}\text { Natural } \\
\text { conceptions }\end{array}$ & Stimulation \\
\hline $\begin{array}{l}\text { Ata } \\
\text { et al., } 2012\end{array}$ & USA & Jan'10-Jul'11 & Retrospective & $\begin{array}{l}\text { Aneuploidy according to } \\
\text { embryos biopsied }\end{array}$ & NA & NA \\
\hline $\begin{array}{l}\text { Labarta } \\
\text { et al., } 2012\end{array}$ & Spain & Sep'06-Mar'10 & $\begin{array}{l}\text { Prospective } \\
\text { crossover study }\end{array}$ & $\begin{array}{l}\text { 1st cycle unstimulated } v s .2 \mathrm{nd} \\
\text { cycle stimulated, same patient }\end{array}$ & Unstimulated & $\begin{array}{l}\text { Stimulated } \\
\text { with } 225 \text { IU }\end{array}$ \\
\hline $\begin{array}{l}\text { Sekhon } \\
\text { et al., } 2017\end{array}$ & USA & Mar'10-Apr'15 & Retrospective & $\begin{array}{l}\text { Cumulative dose and } \\
\text { aneuploidy rate }\end{array}$ & NA & NA \\
\hline $\begin{array}{l}\text { Labarta } \\
\text { et al., } 2017\end{array}$ & Spain & Sep'06-Mar'10 & $\begin{array}{l}\text { Post hoc analysis } \\
\text { of prospective } \\
\text { crossover study }\end{array}$ & $\begin{array}{c}\text { Aneuploidy according to ovarian } \\
\text { response (same-dosage): low } \\
\text { vs. high }\end{array}$ & $\begin{array}{l}\text { Low: }<17 \\
\text { oocytes }\end{array}$ & $\begin{array}{l}\text { High: } \geq 17 \\
\text { oocytes }\end{array}$ \\
\hline $\begin{array}{l}\text { Barash } \\
\text { et al., } 2017\end{array}$ & USA & Jan'13-Jan'13 & Retrospective & $\begin{array}{c}\text { Different dosages of } \\
\text { gonadotropins and aneuploidy } \\
\text { rate }\end{array}$ & NA & NA \\
\hline $\begin{array}{l}\text { Venetis } \\
\text { et al., } 2019\end{array}$ & Australia & Mar'11-Dec'16 & $\begin{array}{l}\text { Retrospective, } \\
\text { Multicenter }\end{array}$ & $\begin{array}{c}\text { Correlation of oocytes retrieved } \\
\text { and the number of euploid } \\
\text { embryos }\end{array}$ & NA & NA \\
\hline $\begin{array}{l}\text { Thorne } \\
\text { et al., } 2019\end{array}$ & USA & Jan'13-Feb'18 & Retrospective & $\begin{array}{c}\text { Final oocyte maturation trigger } \\
\text { rhCG vs. aGnRH }\end{array}$ & rhCG trigger & aGnRH trigger \\
\hline
\end{tabular}

*RCT: randomized controlled trial; IU: international units; USA: United States of America; FSH: follicle stimulating hormone; LH: luteinizing hormone; hMG: human menopausal gonadotropins; NA: not applicable; GnRH: gonadotropin-releasing hormone; rhCG: recombinant human chorionic gonadotropin; aGnRH: agonist of the GnRH; IVF: in vitro fertilization; FISH: Fluorescence in situ hybridization; RIF: repeated implantation failure; IUI: intrauterine insemination; POCs: products of conception; PGT: preimplantation genetic testing; array comparative genomic hybridization; qPCR-based CCS: quantitative polymerase chain reaction - based comprehensive chromosome screening; SNPs: single nucleotide polymorphism; NGS: next-generation sequencing; ITT: intention-to-treat; D3: cleavage-stage embryo.

Triggering of final oocyte maturation: r-hCG vs. GnRH-agonist

One recent study evaluated euploidy rates according to trigger medication. Thorne et al. (2019) compared 366 patients involving 539 cycles (rhCG=336 vs. aG$\mathrm{nRH}=203$ ). Raw analysis showed that the euploidy rate was higher when aGnRH trigger was used $(37.8 \% \pm 2.1 \%$ vs. $30.3 \% \pm 1.8 \%, p=0.01)$. After a multivariate linear regression analysis, trigger medication was no longer predictive of ploidy status [B:-0.02, 95\% CI $(-0.08,0.05), \mathrm{F}$ $(0.19,1), p=0.67]$ (Thorne et al., 2019), with age being the only predictive factor. Of note, in everyday practice, a GnRH trigger is most likely used in younger, hyper-responsive patients, which could explain the differences in baseline characteristics (younger, higher AMH, lower BMI, lower total gonadotropin dose, higher peak estradiol level).

\section{According to Ovarian Response}

Seven non-randomized studies have analyzed whether the aneuploidy rate was associated with the total number of oocytes or embryos generated (Haaf et al., 2009; Rubio et al., 2010; Ata et al., 2012; Barash et al., 2017; Labarta et al., 2017; Sekhon et al., 2017; Venetis et al., 2019). 
Table 1. Continued.

\begin{tabular}{|c|c|c|c|c|c|c|c|}
\hline Author & Inclusion Criteria & Patients & Patients by Group & PGT platform & $\begin{array}{l}\text { Biopsy } \\
\text { Day }\end{array}$ & $\begin{array}{c}\text { Cells } \\
\text { Biopsied }\end{array}$ & Cells Biopsied \\
\hline $\begin{array}{l}\text { Baart } \\
\text { et al., } 2007\end{array}$ & $\begin{array}{c}\text { General IVF } \\
\text { patients, regular } \\
\text { menses, } 5 \text { mill } \\
\text { sperm, 46XX }\end{array}$ & 111 & $\begin{array}{l}\text { Low }=67 \\
\text { High }=44\end{array}$ & $\begin{array}{c}\text { FISH (10 } \\
\text { chromosomes) }\end{array}$ & Day 3 & $\begin{array}{l}\text { Cells } \\
\text { Biopsied }\end{array}$ & $\begin{array}{l}\text { Low }=33.2 \\
\text { High }=34.1\end{array}$ \\
\hline $\begin{array}{l}\text { Weghofer } \\
\text { et al., } 2008\end{array}$ & $\begin{array}{c}\text { General IVF } \\
\text { patients, regular } \\
\text { menses, normal } \\
\text { ovarian function, } \\
46 \mathrm{XX}\end{array}$ & 104 & $\begin{array}{l}\mathrm{FSH}=52 \\
\mathrm{hMG}=52\end{array}$ & $\begin{array}{c}\text { FISH ( } 9 \\
\text { chromosomes) }\end{array}$ & Day 3 & $1-2$ cells & $\begin{array}{l}\mathrm{FSH}=35.0 \\
\mathrm{hMG}=35.9\end{array}$ \\
\hline $\begin{array}{l}\text { Verpoest } \\
\text { et al., } 2008\end{array}$ & $\begin{array}{c}\text { Regular menses, } \\
<3 \text { failed cycles, } \\
\text { normal uterine } \\
\text { cavity }\end{array}$ & 30 & Unstimulated $=30$ & $\begin{array}{c}\text { FISH (5 } \\
\text { chromosomes) }\end{array}$ & Day 3 & 1 cell & 31.4 \\
\hline $\begin{array}{l}\text { Haaf } \\
\text { et al., } 2009\end{array}$ & $\begin{array}{c}>35 \text { years or } \\
\text { previous IVF } \\
\text { cycle with no ET }\end{array}$ & 30 & $\begin{array}{c}\text { <35: } 93 \text { cycles } \\
\text { 35-40: } 376 \text { cycles } \\
\text { >40: } 165 \text { cycles }\end{array}$ & $\begin{array}{c}\text { FISH (5 } \\
\text { chromosomes) }\end{array}$ & Day 0 & 1 cell & NA \\
\hline $\begin{array}{l}\text { Weghofer } \\
\text { et al., } 2009\end{array}$ & $\begin{array}{c}\text { General IVF } \\
\text { patients, regular } \\
\text { menses, normal } \\
\text { ovarian function, } \\
46 \mathrm{XX}\end{array}$ & 104 & $\mathrm{FSH}=52, \mathrm{hMG}=52$ & $\begin{array}{c}\text { FISH (9 } \\
\text { chromosomes) }\end{array}$ & Day 3 & 2 cells & $\begin{array}{l}\mathrm{FSH}=39.8 \\
\mathrm{hMG}=39.6\end{array}$ \\
\hline $\begin{array}{l}\text { Rubio } \\
\text { et al., } 2010\end{array}$ & Oocyte donors & 32 & $\begin{array}{c}\text { First cycle }=32 \\
\text { Second cycle }=22 \\
(10 \text { no biopsy })\end{array}$ & $\begin{array}{c}\text { FISH (9 } \\
\text { chromosomes) }\end{array}$ & Day 3 & 1 cell & 22.0 \\
\hline $\begin{array}{l}\text { Kyrou } \\
\text { et al., } 2011\end{array}$ & $\begin{array}{c}\text { Recurrent } \\
\text { Miscarriage, RIF, } \\
\text { azoospermia } \\
\end{array}$ & 694 & $\begin{array}{c}\text { Agonist }=320 \\
\text { Antagonist }=374\end{array}$ & $\begin{array}{l}\text { FISH (7 Cells } \\
\text { Biopsied) }\end{array}$ & Day 3 & $1-2$ cells & $\begin{array}{c}\text { Agonist }=32.8 \\
\text { Antagonist }=32.7\end{array}$ \\
\hline $\begin{array}{l}\text { Massie } \\
\text { et al., } 2011\end{array}$ & $\begin{array}{c}\text { Infertility } \\
\text { patients trying to } \\
\text { conceive }\end{array}$ & 229 & $\begin{array}{c}\text { No stimulation }=50 \\
\text { FSH for } \mathrm{IUI}=35 \\
\text { FSH for } \mathrm{IVF}=144\end{array}$ & $\begin{array}{l}\text { Trypsin-Wright } \\
\text { G-banding }\end{array}$ & NA & 1 cell & $\begin{array}{c}\text { Natural } 37.0 \\
\text { FSH exposure } \\
37.0 \\
\end{array}$ \\
\hline $\begin{array}{l}\text { Ata } \\
\text { et al., } 2012\end{array}$ & $\begin{array}{c}\text { Infertility patients } \\
\text { undergoing PGT } \\
\text { or Oocyte donors } \\
\end{array}$ & 990 & 7753 embryos & $\begin{array}{l}\text { aCGH-based } \\
\text { CCS }\end{array}$ & $\begin{array}{l}\text { Day 3/ } \\
\text { Day } 5\end{array}$ & POCs & NA \\
\hline $\begin{array}{l}\text { Labarta } \\
\text { et al., } 2012\end{array}$ & Oocyte donors & 232 & $\begin{array}{c}\text { Unstimulated }=185 \\
\text { Stimulated }=47\end{array}$ & $\begin{array}{c}\text { FISH ( } 9 \\
\text { chromosomes) }\end{array}$ & Day 3 & $\begin{array}{l}\text { Day } 3: 1-2 \\
\text { cells; } \\
\text { Day } 5: 3-10 \\
\text { cells }\end{array}$ & 25.4 \\
\hline $\begin{array}{l}\text { Sekhon } \\
\text { et al., } 2017\end{array}$ & $\begin{array}{c}\text { Infertility patients } \\
\text { undergoing PGT }\end{array}$ & 828 & 1122 cycles & $\begin{array}{c}\text { qPCR-based } \\
\text { CCS } \\
\end{array}$ & Day 5 & 1 cell & 38.5 \\
\hline $\begin{array}{l}\text { Labarta } \\
\text { et al., } 2017\end{array}$ & Oocyte donors & 46 & $\begin{array}{l}\text { Low response }=22 \\
\text { High response }=24\end{array}$ & $\begin{array}{c}\text { FISH ( } 9 \\
\text { chromosomes) } \\
\end{array}$ & Day 3 & 4-7 cells & $\begin{array}{l}\text { Low }=25.6 \\
\text { High }=24.9 \\
\end{array}$ \\
\hline $\begin{array}{l}\text { Barash } \\
\text { et al., } 2017\end{array}$ & $\begin{array}{c}\text { Infertility patients } \\
\text { undergoing PGT }\end{array}$ & 681 & 794 cycles & SNP & Day 5 & 4-9 cells & NA \\
\hline $\begin{array}{l}\text { Venetis } \\
\text { et al., } 2019\end{array}$ & $\begin{array}{c}\text { Infertility patients } \\
\text { undergoing PGT }\end{array}$ & 724 & 724 cycles & $\mathrm{aCGH}$ & Day 3 & 1 cell & NA \\
\hline $\begin{array}{l}\text { Thorne } \\
\text { et al., } 2019\end{array}$ & $\begin{array}{l}\text { Infertility patients } \\
\text { undergoing PGT }\end{array}$ & 539 & $\begin{array}{c}\text { hCG trigger }=336 \\
\text { aGnRH } \\
\text { trigger }=203\end{array}$ & $\mathrm{aCGH} / \mathrm{NGS}$ & Day 5 & NA & No restriction \\
\hline
\end{tabular}

The first study correlating ovarian response and aneuploidy was performed in Germany (Haaf et al., 2009), and given that German law does not allow PGT on blastomeres, the authors performed polar body analysis with FISH, and analyzed the aneuploidy rate per cohort size (1-5,6-10, $>10$ oocytes retrieved) and per age $(<35,35-40,>40$ years old). The authors reported that aneuploidy rate increased with increasing age. Interestingly, the aneuploidy rate was similar in each age group and increasing oocyte yield, except in patients < 35years, in which the aneuploidy rate increased with increasing number of oocytes: 1-5 oocytes $23.3 \%$; 6-10 oocytes $34.9 \%$; >10 oocytes $50.9 \%$. Although the authors claim that the increased chromosome error rate may be explained by a non-physiological ovarian stimulation (which interferes with chromosome segregation behavior during maternal meiosis) and that lower oocyte yields may represent a more appropriate response to ovarian stimulation (allowing only the most competent follicles and oocytes to develop and, therefore, reduce oocyte error rates) is important to consider that, when analyzing only patients that reach the biopsy stage or had at least one embryo biopsied, by definition we are 
Table 1. Continued.

\begin{tabular}{|c|c|c|c|c|c|c|}
\hline Author & $\begin{array}{c}\text { Primary Outcome } \\
\text { measure }\end{array}$ & $\begin{array}{l}\text { Oocytes } \\
\text { retrieved }\end{array}$ & $\begin{array}{c}\text { Aneuploidy Rate per } \\
\text { embryo }\end{array}$ & $\begin{array}{l}\text { Aneuploidy } \\
\text { Rate by ITT }\end{array}$ & $\begin{array}{c}\text { Euploid } \\
\text { Embryos (n) }\end{array}$ & $\begin{array}{l}\text { Euploid } \\
\text { Embryos by } \\
\text { ITT (n) }\end{array}$ \\
\hline $\begin{array}{l}\text { Baart } \\
\text { et al., } 2007\end{array}$ & $\begin{array}{l}\text { Oocytes retrieved } \\
\text { Aneuploidy Rate }\end{array}$ & $\begin{array}{l}\text { Low }=8.2 \\
\text { High }=12.1\end{array}$ & $\begin{array}{c}\text { Low }=50 \% \\
\text { High }=62 \%, p=0.48\end{array}$ & $\begin{aligned} \text { Low } & =22 \% \\
\text { High } & =26 \% \\
p & =0.4\end{aligned}$ & $\begin{array}{l}\text { Low }=1.8 \\
\mathrm{High}=1.8\end{array}$ & $\begin{array}{l}\text { Low }=1.4 \\
\mathrm{High}=2.5\end{array}$ \\
\hline $\begin{array}{l}\text { Weghofer } \\
\text { et al., } 2008\end{array}$ & $\begin{array}{l}\text { Oocytes retrieved } \\
\text { Aneuploidy Rate }\end{array}$ & $\begin{array}{l}\mathrm{FSH}=23.7 \\
\mathrm{hMG}=20.3\end{array}$ & $\begin{array}{c}\mathrm{FSH}=54.7 \% \\
\mathrm{hMG}=30.2 \%, p<0.01\end{array}$ & $\begin{array}{l}\text { Only patients } \\
\text { with biopsy } \\
\text { included }\end{array}$ & $\begin{array}{l}\mathrm{FSH}=3.1 \\
\mathrm{hMG}=3.3\end{array}$ & NA \\
\hline $\begin{array}{l}\text { Verpoest } \\
\text { et al., } 2008\end{array}$ & Aneuploidy Rate & $\begin{array}{l}\text { Unstimulated } \\
\quad=0.6\end{array}$ & Unstimulated $=36.4 \%$ & NA & $\begin{array}{l}\text { Unstimulated } \\
\quad=0.2\end{array}$ & NA \\
\hline $\begin{array}{l}\text { Haaf } \\
\text { et al., } 2009\end{array}$ & $\begin{array}{c}\text { Aneuploidy per } \\
\text { cohort size }(1-5,6- \\
10,>10 \text { oocytes }) \\
\text { and per age }(<35 \\
35-40,>40)\end{array}$ & $\begin{array}{l}<35: 8.5 \\
35-40: 8.6 \\
>40: 7.1\end{array}$ & $\begin{array}{c}\text { 1-5 oocytes }(<35: \\
\text { 23.3\%; 35-40:41.2\%; } \\
\text { >40:49.6\%) } \\
6-10 \text { oocytes } \\
(<35: 34.9 \% ; 35-40: \\
43.8 \% ;>40: 50.0 \%) \\
>10 \text { oocytes } \\
(<35: 50.9 \% ; 35- \\
40: 54.6 \% ;>40: 54.1 \%)\end{array}$ & NA & NA & NA \\
\hline $\begin{array}{l}\text { Weghofer } \\
\text { et al., } 2009\end{array}$ & $\begin{array}{l}\text { Oocytes retrieved } \\
\text { Aneuploidy Rate }\end{array}$ & $\begin{array}{l}\mathrm{FSH}=15.2 \\
\mathrm{hMG}=13.8\end{array}$ & $\begin{array}{c}\mathrm{FSH}=70.6 \% \\
\mathrm{hMG}=74.3 \%, \mathrm{NS}\end{array}$ & $\begin{array}{l}\text { NA (only } \\
\text { patients with } \\
\text { biopsy } \\
\text { included) }\end{array}$ & $\begin{array}{l}\mathrm{FSH}=2.1 \\
\mathrm{hMG}=1.9\end{array}$ & NA \\
\hline $\begin{array}{l}\text { Rubio } \\
\text { et al., } 2010\end{array}$ & $\begin{array}{l}\text { Oocytes retrieved } \\
\text { Aneuploidy Rate }\end{array}$ & $\begin{array}{l}\text { Standard }=23.9 \\
\text { Reduced }=14.7\end{array}$ & $\begin{array}{l}\text { Standard }=83.9 \% \\
\text { Reduced }=77.5 \%\end{array}$ & NA & $\begin{array}{l}\text { Standard }=3.1 \\
\text { Reduced }=2.7\end{array}$ & $\begin{array}{l}\text { Standard }=3.1 \\
\text { Reduced }=1.8\end{array}$ \\
\hline $\begin{array}{l}\text { Kyrou } \\
\text { et al., } 2011\end{array}$ & $\begin{array}{l}\text { Oocytes retrieved } \\
\text { Aneuploidy Rate }\end{array}$ & $\begin{array}{c}\text { Agonist }=12.9 \\
\text { Antagonist }=13.6\end{array}$ & $\begin{array}{c}\text { Agonist }=49.9 \% \\
\text { Antagonist } 50.2 \%, \\
p=0.922\end{array}$ & $\begin{array}{l}\text { NA (only } \\
\text { patients with } \\
\text { biopsy } \\
\text { included) }\end{array}$ & $\begin{array}{c}\text { Agonist }=2.7 \\
\text { Antagonist }=3.1\end{array}$ & NA \\
\hline $\begin{array}{l}\text { Massie } \\
\text { et al., } 2011\end{array}$ & Aneuploid gestation & NA & $\begin{array}{c}\text { Natural }=70 \% \\
\text { FSH exposure }=63 \%, \text { NS }\end{array}$ & NA & NA & NA \\
\hline $\begin{array}{l}\text { Ata } \\
\text { et al., } 2012\end{array}$ & $\begin{array}{c}\text { Aneuploidy per } \\
\text { cohort size }(1-4, \\
5-7,8-10,>10 \\
\text { embryos) and per } \\
\text { age }(<35,35-39 \\
40-42, \leq 43)\end{array}$ & NA & $\begin{array}{c}\text { Per age and (D3/ } \\
\text { Blastocyst): } \\
<35(38.3 \% / 61.4 \%) \\
35-39(28.9 \% / 50.27 \%) \\
40-42(16.05 \% \\
/ 33.37 \%) ; \leq 43 \\
(8.95 \% / 17.3 \%)\end{array}$ & NA & NA & NA \\
\hline $\begin{array}{l}\text { Labarta } \\
\text { et al., } 2012\end{array}$ & Aneuploidy Rate & $\begin{array}{c}\text { Unstimulated } \\
\quad=0.8 \\
\text { Stimulated }=17.8\end{array}$ & $\begin{array}{l}\text { Unstimulated }=35.3 \% \\
\text { vs. Stimulated }=40.6 \%\end{array}$ & $\begin{array}{l}\text { Unstimulated } \\
=76.7 \% \\
\text { Stimulated } \\
=40.6 \%\end{array}$ & $\begin{array}{c}\text { Unstimulated } \\
\quad=1.8 \\
\text { Stimulated }=3.9\end{array}$ & $\begin{array}{c}\text { Unstimulated } \\
\quad=1.0 \\
\text { Stimulated }=3.9\end{array}$ \\
\hline $\begin{array}{l}\text { Sekhon } \\
\text { et al., } 2017\end{array}$ & $\begin{array}{l}\text { Cumulative } \\
\text { gonadotropin dose } \\
\text { used, duration of } \\
\text { stimulation }\end{array}$ & NA & $\begin{array}{c}47 \% \\
\text { no association of } \\
\text { cumulative dose and } \\
\text { aneuploidy } \\
\text { (adjusted OR=1.0.49, } \\
p=0.232 \text { ) }\end{array}$ & NA & NA & NA \\
\hline $\begin{array}{l}\text { Labarta } \\
\text { et al., } 2017\end{array}$ & $\begin{array}{c}\text { Number of euploid } \\
\text { embryos }\end{array}$ & $\begin{array}{l}\text { Low }=11.5 \\
\mathrm{High}=23.8\end{array}$ & $\begin{array}{c}\text { Low }=39 \% \\
\text { High }=639 \%, p=0.2\end{array}$ & NA & $\begin{array}{l}\text { Low }=2.7 . \\
\text { High }=5.0\end{array}$ & NA \\
\hline $\begin{array}{l}\text { Barash } \\
\text { et al., } 2017\end{array}$ & $\begin{array}{l}\text { Aneuploidy Rate } \\
\text { per dosage and per } \\
\text { age }\end{array}$ & 16,3 & $\begin{array}{c}<35 \text { years old: } 62.86 \% \\
35-37 \text { years old: } \\
55.75 \% \\
38-40 \text { years old: } \\
42.23 \% \\
>41 \text { years old: } 27.53 \%\end{array}$ & NA & NA & NA \\
\hline $\begin{array}{l}\text { Venetis } \\
\text { et al., } 2019\end{array}$ & $\begin{array}{c}\text { Number of euploid } \\
\text { embryos }\end{array}$ & $\begin{array}{c}11.7(11.1- \\
12.2)\end{array}$ & $\begin{array}{c}\text { Euploidy } 17.4 \% \\
\text { (15.5-19.3), Aneuploidy } \\
82.6 \%\end{array}$ & NA & $1.0(0.9-1.1)$ & NA \\
\hline $\begin{array}{l}\text { Thorne } \\
\text { et al., } 2019\end{array}$ & Aneuploidy Rate & $\begin{array}{c}\mathrm{hCG}=11.5 \\
\mathrm{aGnRH}=19.3\end{array}$ & $\begin{array}{c}\text { hCG }=30.3 \% \text { vs. } \\
\text { aGnRH }=37.8 \% \\
\text { MLRA: no differences } \\
\text { between groups }\end{array}$ & NA & NA & NA \\
\hline
\end{tabular}


excluding patients who are at the higher risk of producing an embryonic aneuploidy: poor/low responder patients who are generally older or with low quality oocytes and/ or embryos. With these in mind, most of the patients who would have had between 1-5 oocytes retrieved with an abnormal oocyte did not reach biopsy and analysis.

Rubio et al. (2010) performed a sub-analysis comparing patients who after two cycles, first under 225UI then a second cycle under $150 \mathrm{UI}$, responded with similarly number of oocytes retrieved. Although the authors found higher fertilization rates ( $82.3 \%$ vs. $71.4 \%)$ with the reduced dose, the proportion of euploid blastocysts per MII retrieved $(11.4 \%$ vs. $20.2 \%, p>0.05)$ and the mean euploid blastocysts per donor (1.5 vs. 1.5, $p>0.05)$ were similar in both groups (Rubio et al., 2010).

Next, Ata et al. (2012) ran a retrospective analysis under aCGH including 1218 blastocysts from 203 women, with results stratified by age and number of embryos generated. The euploidy rates were comparable within each age group ( $<35,35-39,40-42, \geq 43$ ), but decreased with increasing age: $1-4$ blastocysts: $70.2 \%, 66.0 \%, 49.1 \%$, $34.2 \%, 16.7 \%$, respectively; 5-7 blastocysts: $77.5 \%$, $69.9 \%, 52.3 \%, 31.0 \%, 13.3 \%$, respectively; $8-10$ blastocysts: $62.4 \%, 56.7 \%, 48.3 \%, 27.4 \% 22.5 \%$, respectively; and $>10$ blastocysts $66.7 \%, 53.3 \%, 51.4 \%, 40.9 \%$, $16.7 \%$, respectively (Ata et al., 2012). A linear regression analysis showed that for every 1-year increase in age, the euploidy rate decreased $2.9 \%$, and a logistic regression analysis revealed that the odds of having at least one euploid embryo was significantly decreased by female age: OR 0.82 (95\% CI 0.70-0.94, $p=0.006)$, interpreted as $28 \%$ less likely with each extra year. Nevertheless, the odds of having at least one euploid embryo increase by every additional embryo available: OR 1.55 (95\% CI $1.25-$ $1.93), p<0.001$, interpreted as 1.5 times more likely with each extra embryo (Ata et al., 2012).

Subsequently, Sekhon et al. (2017) ran a retrospective study using a logistic regression fit with generalized estimating equations (GEE) for large data analysis, stratifying patients by treatment protocol, duration of stimulation, and by total gonadotropin dose. After controlling for several factors (age, $\mathrm{FSH}$, follicle count, protocol, days of stimulation protocol, and diagnosis, there was no significant association between cumulative dose of gonadotropins and the likelihood of aneuploidy (adjusted OR 1.049, $p=0.232$ ). Nevertheless, if more than 12 days of stimulation were necessary, a $20 \%$ increase in the likelihood of aneuploidy was found (adjusted OR 1.20, 95\% CI 1.125-1.282, $p<0.001$ ), with an extra $16.4 \%$ increase in the odds of aneuploidy with every 1000 UI increase (adjusted OR 1.164, $p=0.002$ ) (Sekhon et al., 2017). These data could suggest a susceptibility to low oocyte quality, which is often seen in ageing patients and/or patients with low ovarian reserve.

Subsequently, Labarta et al. (2017) performed a posthoc analysis of a prospective cohort study from 2012 (Labarta et al., 2012). The authors reported that when the number of oocytes obtained was $\geq 17$ oocytes, the mean number of euploid embryos was 5.0, while it was 2.6 when the ovarian response was lower $(p<0.001)$. The authors found that the number of euploid embryos was inversely related to the ovarian sensitivity index, a correlation of the amount of gonadotropins required per oocyte retrieved (Huber et al., 2013), reflecting better ovarian competence. The authors concluded that a high ovarian response through moderate doses of gonadotropin in young normo-ovulatory women does not increase the rate of embryonic aneuploidy (Labarta et al., 2017).

Barash et al. (2017) analyzed whether different doses of gonadotropins influence euploidy rates. The authors performed a retrospective study of SNPs/PGT outcome data on blastocysts biopsied on D5/D6, from a total of 4034 embryos analyzed. The authors conducted a retrospective study of 4034 embryos on D5/D6 analyzed by SNPs. The cycles were analyzed by total gonadotropin dose $(<3000$ IU, 3000-5000 IU and $>5000$ IU), by retrieved oocytes $(1-5,5-10,10-15$ and $>15$ oocytes) and by patient age $(<35,35-37,38-40$ and $\geq 41$ years). Euploidy rates within the same age group were reported to be similar, regardless of total dose used and/or oocytes retrieved. Furthermore, the euploidy rates were not affected by high doses of gonadotropin (450 IU per day) in patients $<38$ years. In the group of patients $\geq 38$ years, the authors found a gradual decrease in euploidy rates when high doses were used, although it only reached statistical significance in patients aged 38 to 40 years (Barash et al., 2017). Interestingly, within each age group, the usable blastocyst rate was similar when $<3000$ IU gonadotropins were used in cycles where more than 5000 IU were used. The authors observed a difference in usable blastocyst rate, with increasing age, although it only reached statistical significance in patients aged 35 to 37 years (OR $=1.417,95 \% \mathrm{CI}$ 1.047-1.918, $p=0.024)$.

Lastly, Venetis et al. (2019) analyzed 724 patients using D3 biopsy and aCGH and included all significant population and stimulation characteristics as covariates in a multivariate regression GEE, as well as an interaction term between female age and number of oocytes, revealed that female age (coefficient: $-0.06,95 \%$ CI -0.11 to -0.016 , $p=0.009$ ) and the number of oocytes retrieved (coefficient: $+0.40 ; 95 \%$ CI $0.24-0.56, p<0.001$ ) remain the only significant prognosticators of the total number of euploid embryos.

Among the studies associating ovarian response to aneuploidy, only Haaf et al. (2009) reported an increased aneuploidy rate, although there were important statistical limitations. Overall, all other 6 studies reported similar results, concluding that ovarian response did not alter euploidy rate or the total number of euploid embryos. Moreover, a case can be made towards better results with increasing number of biopsied embryos.

\section{DISCUSSION}

This systematic review investigated a topic of great importance for reproductive endocrinologists in the field of reproductive medicine, and, to our knowledge, it is the first of its kind. Concerning the impact of COS on chromosome imbalance in human embryos, the current review did not find a clear correlation. On one hand, it has been previously proved that natural cycles are also associated with aneuploidy, but on the other hand, it seems that more intensive stimulations are indeed associated to a higher proportion of aneuploidy. Nevertheless, given that a higher response is associated with more oocytes retrieved, this translates into potentially more euploid embryos available for transfer.

Up to the present time, the only factor incontrovertibly linked to human aneuploidy is increasing maternal age (Franasiak et al., 2014). Even though chromosomal errors seem to be linked to a generalized oocyte disorganization rather than to a single/specific oocyte alteration, there is increasing evidence supporting the fact that each aneuploidy event is not dependent on a specific variable, but on a group of variables. To date, it has been impossible to isolate if the gonadotropin dosage actually distresses the oocyte or if the aneuploidy is simply a reflection of its low quality. If, for a moment, we assume that COS does, in fact, impact embryonic aneuploidy, what would it be preferable to have, a lower ratio of aneuploidy or higher total number of euploid embryos? Morin et al. (2014) published that in patients with $\leq 3$ euploid blastocysts, clinical PR was higher in double, compared to single embryo transfers; 
however, in patients with $\geq 4$ euploid blastocysts, clinical PR was not decreased with single embryo transfer. These findings support the hypothesis that euploid embryo cohort size is an important prognosis factor in ART treatment, and the number of euploid embryos has to be taken into account, more significantly than the ratio of euploid/aneuploid embryos (Morin et al., 2014; Labarta et al., 2017).

One particular strength is the detailed analysis on a per study basis and grouping studies with similar intervention criteria. Secondly, whenever possible, the authors ran an ITT analysis, with the purpose of homogenizing the denominator in the analysis groups

Limitations of the present systematic review should be acknowledged. First, only one RCT has been performed. Even though the quality of the rest of the studies is high (NOS above 7), bias associated with retrospective studies cannot be ruled out. Second, the studies included presented a large diversity in the assessment of embryonic aneuploidy, and, given the great heterogeneity between studies, a meta-analysis could not be performed. Important differences consisted in the different platforms used to analyze aneuploidy and the embryo stage at which the biopsy was performed. Third, in those studies correlating ovarian response with aneuploidy, no information regarding the number of aneuploid embryos and the number of embryos biopsied was reported, which prevented pooling the data. In this regard, an effort was made to contact corresponding authors, but it was not possible to obtain the full data. And fourth, this review did not address the problem of mosaicism (Munné et al., 2002; Cram et al., 2019). For the purpose of this review, all embryonic abnormalities were considered as aneuploidy, mainly because that is how abnormalities were reported in the studies included; therefore, mosaicism was included as an aneuploid embryo when reported. Recently, the genomic technologies available have improved in sensitivity and resolution and have permitted a more complete spectrum of chromosome abnormalities to be identified, but long-term studies are needed to understand the clinical impact of mosaicism. In our opinion, whether mosaicism is considered separately or together with aneuploidy, does not changes the analysis, as it is still considered to be an abnormal result and associated to lower clinical outcomes (Munné et al., 2020).

Remarkably, no large randomized clinical trial on this topic has yet been conducted. Further evidence is needed to ascertain if there is a negative impact of COS, especially at the cellular level. Furthermore, biomarkers to predict minimal effective dose to achieve the highest reproductive potential are needed.

\section{AUTHORS' CONTRIBUTIONS}

J.R-P. conceptualized the review. J.R-P and E.C-B. searched databases, selected papers and performed data extraction and analysis. J.R-P. provided statistical support and data analysis. J.R-P. took the lead in writing the review. M-J.G. and E.C-B. revised several draft versions of the manuscript. All authors read and approved the final version of the manuscript.

PROSPERO registration number: CRD42019120803

\section{CONFLICT OF INTERESTS}

The author(s) declared no potential conflicts of interest with respect to the study, authorship, and/or publication of this article.

\section{Corresponding author:}

Jorge Rodriguez-Purata

Clinica de la Fertilidad "CdelaF"

Colonia Santa Fe, Cuajimalpa de Morelos
Mexico City, Mexico

E-mail: jorge@cdelaf.com

ORCID ID: orcid.org/0000-0001-5432-0884

\section{REFERENCES}

Ata B, Kaplan B, Danzer H, Glassner M, Opsahl M, Tan SL, Munné S. Array $\mathrm{CGH}$ analysis shows that aneuploidy is not related to the number of embryos generated. Reprod Biomed Online. 2012;24:614-20. PMID: 22503277 DOI: 10.1016/j.rbmo.2012.02.009

Baart EB, Martini E, Eijkemans MJ, Van Opstal D, Beckers NGM, Verhoeff A, Macklon NS, Fauser BCJM. Milder ovarian stimulation for in-vitro fertilization reduces aneuploidy in the human preimplantation embryo: a randomized controlled trial. Hum Reprod. 2007;22:980-8. PMID: 17204525 DOI: $10.1093 /$ humrep/del484

Barash OO, Hinckley MD, Rosenbluth EM, Ivani KA, Weckstein LN. High gonadotropin dosage does not affect euploidy and pregnancy rates in IVF PGS cycles with single embryo transfer. Hum Reprod. 2017;32:2209-17. PMID: 29040519 DOI: 10.1093/humrep/dex299

Barlow AL, Hultén MA. Combined immunocytogenetic and molecular cytogenetic analysis of meiosis I oocytes from normal human females. Zygote. 1998;6:27-38. PMID: 9652069 DOI: $10.1017 /$ S0967199400005050

Capalbo A, Bono S, Spizzichino L, Biricik A, Baldi M, Colamaria S, Ubaldi FM, Rienzi L, Fiorentino F. Sequential comprehensive chromosome analysis on polar bodies, blastomeres and trophoblast: insights into female meiotic errors and chromosomal segregation in the preimplantation window of embryo development. Hum Reprod. 2013;28:50918. PMID: 23148203 DOI: 10.1093/humrep/des394

Champion MD, Hawley RS. Playing for half the deck: the molecular biology of meiosis. Nat Cell Biol. 2002;4:50-6. PMID: 12479615 DOI: $10.1038 / n c b-n m$-fertilityS50

Cram DS, Leigh D, Handyside A, Rechitsky L, Xu K, Harton G, Grifo J, Rubio C, Fragouli E, Kahraman S, Forman E, Katz-Jaffe M, Tempest H, Thornhill A, Strom C, Escudero T, Qiao J, Munne S, Simpson JL, Kuliev A. PGDIS Position Statement on the Transfer of Mosaic Embryos 2019. Reprod Biomed Online. 2019;39:e1-e4. PMID: 31421710 DOI: $10.1016 /$ j.rbmo.2019.06.012

De Rycke M, Belva F, Goossens V, Moutou C, SenGupta SB, Traeger-Synodinos J, Coonen E. ESHRE PGD Consortium data collection XIII: cycles from January to December 2010 with pregnancy follow-up to October 2011. Hum Reprod. 2015;30:1763-89. PMID: 26071418 DOI: 10.1093/ humrep/dev 122

Delhanty JD. Mechanisms of aneuploidy induction in human oogenesis and early embryogenesis. Cytogenet Genome Res. 2005;111:237-44. PMID: 16192699 DOI: $10.1159 / 000086894$

Drakopoulos P, Blockeel C, Stoop D, Camus M, de Vos M, Tournaye $\mathrm{H}$, Polyzos NP. Conventional ovarian stimulation and single embryo transfer for IVF/ICSI. How many oocytes do we need to maximize cumulative live birth rates after utilization of all fresh and frozen embryos? Hum Reprod. 2016;31:370-6. PMID: 26724797 DOI: 10.1093/ humrep/dev316 
Edgar DH, Whalley KM, Mills JA. Effects of high-dose and multiple-dose gonadotropin stimulation on mouse oocyte quality as assessed by preimplantation development following in vitro fertilization. J In Vitro Fert Embryo Transf. 1987;4:273-6. PMID: 3694007 DOI: 10.1007/BF01555203

Elbling L, Colot M. Abnormal development and transport and increased sister-chromatid exchange in preimplantation embryos following superovulation in mice. Mutat Res. 1985;147:189-95. PMID: 4022033 DOI: 10.1016/0165$1161(85) 90057-3$

Fragouli E, Alfarawati S, Spath K, Jaroudi S, Sarasa J, Enciso $M$, Wells $D$. The origin and impact of embryonic aneuploidy. Hum Genet. 2013;132:1001-13. PMID: 23620267 DOI: $10.1007 / \mathrm{s} 00439-013-1309-0$

Franasiak JM, Forman EJ, Hong KH, Werner MD, Upham KM, Treff NR, Scott RT Jr. The nature of aneuploidy with increasing age of the female partner: a review of 15,169 consecutive trophectoderm biopsies evaluated with comprehensive chromosomal screening. Fertil Steril. 2014;101:656-63.e1. PMID: 24355045 DOI: $10.1016 /$ j.fertnstert.2013.11.004

Frumkin T, Malcov M, Yaron Y, Ben-Yosef D. Elucidating the origin of chromosomal aberrations in IVF embryos by preimplantation genetic analysis. Mol Cell Endocrinol. 2008;282:112-9. PMID: 18177997 DOI: 10.1016/j.mce.2007.11.009

Golbus MS. The influence of strain, maternal age, and method of maturation on mouse oocyte aneuploidy. Cytogenet Genome Res. 1981;31:84-90. DOI: 10.1159/000131629

Haaf T, Hahn A, Lambrecht A, Grossmann B, Schwaab E, Khanaga O, Hahn T, Tresch A, Schorsch M. A high oocyte yield for intracytoplasmic sperm injection treatment is associated with an increased chromosome error rate. Fertil Steril. 2009;91:733-8. PMID: 18314124 DOI: 10.1016/j. fertnstert.2008.01.012

Handyside AH, Pattinson JK, Penketh RJ, Delhanty JD, Winston RM, Tuddenham EG. Biopsy of human preimplantation embryos and sexing by DNA amplification. Lancet. 1989;1:347-9. PMID: 2464730 DOI: 10.1016/S01406736(89)91723-6

Hartling L, Hamm M, Milne A, Vandermeer B, Santaguida PL, Ansari M, Tsertsvadze A, Hempel S, Shekelle P, Dryden DM. Validity and Inter-Rater Reliability Testing of Quality Assessment Instruments [Internet]. Rockville (MD): Agency for Healthcare Research and Quality (US); 2012. Report No: 12-EHC039-EF.

Hassold T, Hunt P. To err (meiotically) is human: the genesis of human aneuploidy. Nat Rev Genet. 2001;2:280-91. PMID: 11283700 DOI: $10.1038 / 35066065$

Hodges CA, Ilagan A, Jennings D, Keri R, Nilson J, Hunt PA. Experimental evidence that changes in oocyte growth influence meiotic chromosome segregation. Hum Reprod. 2002;17:117180. PMID: 11980735 DOI: 10.1093/humrep/17.5.1171

Huber M, Hadziosmanovic N, Berglund L, Holte J. Using the ovarian sensitivity index to define poor, normal, and high response after controlled ovarian hyperstimulation in the long gonadotropin-releasing hormone-agonist protocol: suggestions for a new principle to solve an old problem. Fertil Steril. 2013;100:1270-6.e3. PMID: 23931964 DOI: 10.1016/j.fertnstert.2013.06.049
Jackson KV, Ginsburg ES, Hornstein MD, Rein MS, Clarke RN. Multinucleation in normally fertilized embryos is associated with an accelerated ovulation induction response and lower implantation and pregnancy rates in in vitro fertilization-embryo transfer cycles. Fertil Steril. 1998;70:606. PMID: 9660422 DOI: 10.1016/S0015-0282(98)00100-9

Kyrou D, Verpoest W, Staessen C, De Vos A, Haentjens P, Liebaers I, Devroey $P$. No relationship between the type of pituitary suppression for IVF and chromosomal abnormality rates of blastomeres: an observational study. Fertil Steril. 2011;95:563-7. PMID: 20576261 DOI: 10.1016/j. fertnstert.2010.05.020

Labarta E, Bosch E, Alamá P, Rubio C, Rodrigo L, Pellicer $A$. Moderate ovarian stimulation does not increase the incidence of human embryo chromosomal abnormalities in in vitro fertilization cycles. J Clin Endocrinol Metab. 2012;97:E1987-94. PMID: 22865900 DOI: 10.1210/ jc. $2012-1738$

Labarta E, Bosch E, Mercader A, Alamá P, Mateu E, Pellicer A. A Higher Ovarian Response after Stimulation for IVF Is Related to a Higher Number of Euploid Embryos. Biomed Res Int. 2017;2017:5637923.

Macklon NS, Stouffer RL, Giudice LC, Fauser BC. The Science behind 25 Years of ovarian stimulation for in vitro fertilization. Endocr Rev. 2006;27:170-207. PMID: 16434510 DOI: $10.1210 /$ er.2005-0015

Massie JA, Shahine LK, Milki AA, Westphal LM, Lathi RB. Ovarian stimulation and the risk of aneuploid conceptions. Fertil Steril. 2011;95:970-2. PMID: 20828683 DOI: 10.1016/j.fertnstert.2010.07.1088

Moher D, Liberati A, Tetzlaff J, Altman DG; PRISMA Group. Preferred reporting items for systematic reviews and meta-analyses: the PRISMA statement. J Clin Epidemiol. 2009;62:1006-12. PMID: 19631508 DOI: $10.1016 / \mathrm{j}$. jclinepi.2009.06.005

Morin S, Melzer-Ross K, McCulloh D, Grifo J, Munné S. A greater number of euploid blastocysts in a given cohort predicts excellent outcomes in single embryo transfer cycles. J Assist Reprod Genet. 2014;31:667-73. PMID: 24659020 DOI: $10.1007 /$ s10815-014-0217-0

Munne S, Magli C, Adler A, Wright G, Boer K de, Mortimer D, Tucker M, Cohen J, Gianaroli L. Treatment-related chromosome abnormalities in human embryos. Hum Reprod. 1997;12:780-4. PMID: 9159442 DOI: 10.1093/ humrep/12.4.780

Munné S, Sandalinas M, Escudero T, Márquez C, Cohen J. Chromosome mosaicism in cleavage-stage human embryos: evidence of a maternal age effect. Reprod Biomed Online. 2002;4:223-32. PMID: 12709271 DOI: 10.1016/ S1472-6483(10)61810-X

Munné S, Kaplan B, Frattarelli JL, Child T, Nakhuda G, Shamma FN, Silverberg K, Kalista T, Handyside AH, KatzJaffe M, Wells D, Gordon T, Stock-Myer S, Willman S; STAR Study Group. Preimplantation genetic testing for aneuploidy versus morphology as selection criteria for single frozen-thawed embryo transfer in good-prognosis patients: a multicenter randomized clinical trial. Fertil Steril. 2019;112:1071-9.e7. PMID: 31551155 DOI: 10.1016/j. fertnstert.2019.07.1346 
Munné S, Spinella F, Grifo J, Zhang J, Beltran MP, Fragouli $E$, Fiorentino $F$. Clinical outcomes after the transfer of blastocysts characterized as mosaic by high resolution Next Generation Sequencing- further insights. Eur J Med Genet. 2020;63:103741 PMID: 31445143 DOI: 10.1016/j. ejmg.2019.103741

Nagaoka SI, Hassold TJ, Hunt PA. Human aneuploidy: mechanisms and new insights into an age-old problem. Nat Rev Genet. 2012;13:493-504. PMID: 22705668 DOI: $10.1038 / \mathrm{nrg} 3245$

Nicolaidis $P$, Petersen MB. Origin and mechanisms of non-disjunction in human autosomal trisomies. Hum Reprod Oxf Engl. 1998;13:313-9. PMID: 9557829 DOI: 10.1093/humrep/13.2.313

Obradors A, Rius M, Cuzzi J, Daina G, Gutiérrez-Mateo C, Pujol A, Marina F, Márquez C, Benet J, Navarro J. Errors at mitotic segregation early in oogenesis and at first meiotic division in oocytes from donor females: comparative genomic hybridization analyses in metaphase II oocytes and their first polar body. Fertil Steril. 2010;93:675-9. PMID: 19878936 DOI: $10.1016 /$ j.fertnstert.2009.08.050

Practice Committees of the American Society for Reproductive Medicine and the Society for Assisted Reproductive Technology. The use of preimplantation genetic testing for aneuploidy (PGT-A): a committee opinion. Fertil Steril. $2018 ; 109: 429-36$. PMID: 29566854. DOI: $10.1016 / \mathrm{j}$. fertnstert.2018.01.002

Qi ST, Liang LF, Xian YX, Liu JQ, Wang W. Arrested human embryos are more likely to have abnormal chromosomes than developing embryos from women of advanced maternal age. J Ovarian Res. 2014;7:65. PMID: 24955133 DOI: $10.1186 / 1757-2215-7-65$

Rodriguez-Purata J, Lee J, Whitehouse M, Moschini RM, Knopman J, Duke M, Sandler B, Copperman A. Embryo selection versus natural selection: how do outcomes of comprehensive chromosome screening of blastocysts compare with the analysis of products of conception from early pregnancy loss (dilation and curettage) among an assisted reproductive technology population? Fertil Steril. 2015;104:1460-66.e1-12. PMID: 26361205 DOI: $10.1016 /$ j.fertnstert.2015.08.007

Rodriguez-Purata J, Lee J, Whitehouse M, Duke M, Grunfeld L, Sandler B, Copperman A, Mukherjee T. Reproductive outcome is optimized by genomic embryo screening, vitrification, and subsequent transfer into a prepared synchronous endometrium. J Assist Reprod Genet. 2016;33:40112. PMID: 26768140 DOI: $10.1007 /$ s10815-016-0647-y

Rubio C, Mercader A, Alamá P, Lizán C, Rodrigo L, Labarta E, Melo M, Pellicer A, Remohí J. Prospective cohort study in high responder oocyte donors using two hormonal stimulation protocols: impact on embryo aneuploidy and development. Hum Reprod. 2010;25:2290-7. PMID: 20627979 DOI: $10.1093 /$ humrep/deq174

Santaló J, Estop AM, Egozcue J. The chromosome complement of first-cleavage mouse embryos after in vitro fertilization. J In Vitro Fert Embryo Transf. 1986;3:99-105. PMID: 3701186 DOI: 10.1007/BF01139354
Sato A, Otsu E, Negishi H, Utsunomiya T, Arima T. Aberrant DNA methylation of imprinted loci in superovulated oocytes. Hum Reprod. 2007;22:26-35. PMID: 16923747 DOI: $10.1093 /$ humrep/del316

Sato F, Marrs RP. The effect of pregnant mare serum gonadotropin on mouse embryos fertilized in vivo or in vitro. J In Vitro Fert Embryo Transf. 1986;3:353-7. PMID: 3805852 DOI: $10.1007 / B F 01133247$

Schardt C, Adams MB, Owens T, Keitz S, Fontelo P. Utilization of the PICO framework to improve searching PubMed for clinical questions. BMC Med Inform Decis Mak. 2007;7:16. PMID: 17573961 DOI: $10.1186 / 1472-6947-7-16$

Scott RT Jr, Upham KM, Forman EJ, Zhao T, Treff NR. Cleavage-stage biopsy significantly impairs human embryonic implantation potential while blastocyst biopsy does not: a randomized and paired clinical trial. Fertil Steril. 2013;100:624-30. PMID: 23773313 DOI: 10.1016/j.fertnstert.2013.04.039

Sedgwick P. Intention to treat analysis versus per protocol analysis of trial data. BMJ. 2015;350:h681. PMID: 25663096 DOI: $10.1136 / \mathrm{bmj} . \mathrm{h} 681$

Sekhon L, Shaia K, Santistevan A, Cohn KH, Lee JA, Beim $P Y$, Copperman $A B$. The cumulative dose of gonadotropins used for controlled ovarian stimulation does not influence the odds of embryonic aneuploidy in patients with normal ovarian response. J Assist Reprod Genet. 2017;34:749-58. PMID: 28321529 DOI: 10.1007/s10815-017-0909-3

Sermon K, Capalbo A, Cohen J, Coonen E, De Rycke M, De Vos A, Delhanty J, Fiorentino F, Gleicher N, Griesinger G, Grifo J, Handyside A, Harper J, Kokkali G, Mastenbroek S, Meldrum $D$, Meseguer M, Montag M, Munné S, Rienzi L, et al. The why, the how and the when of PGS 2.0: current practices and expert opinions of fertility specialists, molecular biologists, and embryologists. Mol Hum Reprod. 2016;22:845-57 PMID: 27256483 DOI: 10.1093/molehr/gaw034

Taylor TH, Gitlin SA, Patrick JL, Crain JL, Wilson JM, Griffin DK. The origin, mechanisms, incidence and clinical consequences of chromosomal mosaicism in humans. Hum Reprod Update. 2014;20:571-81. PMID: 24667481 DOI: 10.1093/humupd/dmu016

Tease C, Hartshorne GM, Hultén MA. Patterns of meiotic recombination in human fetal oocytes. Am J Hum Genet. 2002;70:1469-79. PMID: 11992253 DOI: $10.1086 / 340734$

Thorne J, Loza A, Kaye L, Nulsen J, Benadiva C, Grow D, Engmann L. Euploidy rates between cycles triggered with gonadotropin-releasing hormone agonist and human chorionic gonadotropin. Fertil Steril. 2019;112:258-65. PMID: 31103285 DOI: $10.1016 /$ j.fertnstert.2019.03.040

Trounson AO, Leeton JF, Wood C, Webb J, Wood J. Pregnancies in humans by fertilization in vitro and embryo transfer in the controlled ovulatory cycle. Science. $1981 ; 212: 681-$ 2. PMID: 7221556 DOI: $10.1126 /$ science. 7221557

Venetis CA, Tilia L, Panlilio E, Kan A. Is more better? A higher oocyte yield is independently associated with more day3 euploid embryos after ICSI. Hum Reprod. 2019;34:7983. PMID: 30476100 DOI: 10.1093/humrep/dey342 
Verberg MF, Macklon NS, Nargund G, Frydman R, Devroey $P$, Broekmans FJ, Fauser BC. Mild ovarian stimulation for IVF. Hum Reprod Update. 2009;15:13-29. PMID: 19091755 DOI: 10.1093/humupd/dmn056

Verpoest W, Fauser BC, Papanikolaou E, Staessen C, Van Landuyt L, Donoso P, Tournaye H, Liebaers I, Devroey P. Chromosomal aneuploidy in embryos conceived with unstimulated cycle IVF. Hum Reprod. 2008;23:2369-71. PMID: 18621755 DOI: 10.1093/humrep/den269

Vogel R, Spielmann H. Genotoxic and embryotoxic effects of gonadotropin-hyperstimulated ovulation of murine oocytes, preimplantation embryos, and term fetuses. Reprod Toxicol Elmsford N. 1992;6:329-33. PMID: 1521006 DOI: 10.1016/0890-6238(92)90196-Z
Weghofer A, Munné S, Brannath W, Chen S, Tomkin G, Cekleniak N, Garrisi M, Barad D, Cohen J, Gleicher N. The impact of $\mathrm{LH}$-containing gonadotropins on diploidy rates in preimplantation embryos: long protocol stimulation. Hum Reprod. 2008;23:499-503. PMID: 18182396 DOI: 10.1093 /humrep/dem412

Weghofer A, Munné S, Brannath W, Chen S, Barad D, Cohen J, Gleicher N. The impact of LH-containing gonadotropin stimulation on euploidy rates in preimplantation embryos: antagonist cycles. Fertil Steril. 2009;92:937-42. PMID: 18774557 DOI: $10.1016 / \mathrm{j}$.

fertnstert.2008.07.1735 


\begin{tabular}{|c|c|c|c|c|c|c|}
\hline PATIENTS & & INTERVENTION & & OUTCOME & & COMPARISON \\
\hline $\begin{array}{l}\text { "Ovulation } \\
\text { Induction" OR } \\
\text { "ovarian } \\
\text { stimulation" OR } \\
\text { COS OR } \\
\text { "Ovarian } \\
\text { hyperstimulation" } \\
\text { OR } \\
\text { COH }\end{array}$ & AND & $\begin{array}{l}\text { "Preimplantation Diagnosis" OR } \\
\text { "preimplantation genetic } \\
\text { screening" } \\
\text { OR } \\
\text { PGS } \\
\text { OR } \\
\text { "preimplantation genetic testing" } \\
\text { OR } \\
\text { PGT OR } \\
\text { "preimplantation genetic } \\
\text { diagnosis" OR } \\
\text { PGD OR } \\
\text { "genetic screening" OR } \\
\text { "genetic testing" OR } \\
\text { "comprehensive chromosome } \\
\text { screening" OR } \\
\text { "comprehensive chromosome } \\
\text { analysis" OR } \\
\text { "next-generation sequencing" OR } \\
\text { NGS OR } \\
\text { "comparative genomic } \\
\text { hybridization" OR } \\
\text { CGH OR } \\
\text { "array comparative genomic } \\
\text { hybridization" OR } \\
\text { aCGH OR } \\
\text { "single nucleotide polymorphism" } \\
\text { OR } \\
\text { "Polymorphism, Single Nucleotide" } \\
\text { OR } \\
\text { SNP OR } \\
\text { "polymerase chain reaction" OR } \\
\text { PCR OR } \\
\text { "quantitative polymerase chain } \\
\text { reaction" OR } \\
\text { qPCR OR } \\
\text { "whole genome sequencing" OR } \\
\text { "microarray analysis" }\end{array}$ & AND & $\begin{array}{l}\text { Aneuploidy OR } \\
\text { aneuploid* OR } \\
\text { euploidy OR } \\
\text { euploid* OR } \\
\text { "chromosome } \\
\text { disorders" OR } \\
\text { "chromosome } \\
\text { aberrations/ } \\
\text { chemically } \\
\text { induced" }\end{array}$ & AND & $\begin{array}{l}\text { "Fertilization } \\
\text { in Vitro" OR } \\
\text { "in vitro } \\
\text { fertilization" OR } \\
\text { IVF OR } \\
\text { "Reproductive } \\
\text { Techniques, } \\
\text { Assisted" }\end{array}$ \\
\hline
\end{tabular}


Supplemental Table 2. Characteristics and Newcastle-Ottawa scale score of studies included in a systematic review of the association of ovarian stimulation with embryonic aneuploidy in in vitro fertilization cycles coupled with for preimplantation genetic testing.

\begin{tabular}{|c|c|c|c|c|c|c|c|c|c|}
\hline \multirow[b]{2}{*}{ Author } & \multicolumn{4}{|c|}{ Selection } & \multirow{2}{*}{$\begin{array}{c}\text { Compa- } \\
\text { rability } \\
\\
\text { Com- } \\
\text { para- } \\
\text { bility of } \\
\text { Cohorts }\end{array}$} & \multicolumn{3}{|c|}{ Outcome } & \multirow[b]{2}{*}{$\begin{array}{c}\text { Quality } \\
\text { Score }\end{array}$} \\
\hline & $\begin{array}{c}\text { Repre- } \\
\text { senta- } \\
\text { tiveness } \\
\text { Quality } \\
\text { Score of } \\
\text { Exposed } \\
\text { Cohort }\end{array}$ & $\begin{array}{c}\text { of the } \\
\text { Non- } \\
\text { Exposed } \\
\text { Cohort } \\
\text { from } \\
\text { Same } \\
\text { Source as } \\
\text { Exposed } \\
\text { Cohort }\end{array}$ & $\begin{array}{l}\text { Ascertain- } \\
\text { ment of } \\
\text { Exposure }\end{array}$ & $\begin{array}{c}\text { Outcome } \\
\quad \text { of } \\
\text { Interest } \\
\text { Was Not } \\
\text { Present } \\
\text { at Start of } \\
\text { Study }\end{array}$ & & $\begin{array}{l}\text { Assess- } \\
\text { ment of } \\
\text { Outcome }\end{array}$ & $\begin{array}{l}\text { Follow } \\
\text {-Up Long } \\
\text { Enough } \\
\text { for } \\
\text { Outcome } \\
\text { to Occur }\end{array}$ & $\begin{array}{c}\text { Adequacy } \\
\text { of Follow } \\
\text {-Up }\end{array}$ & \\
\hline $\begin{array}{l}\text { Baart } \\
\text { et al., } 2007\end{array}$ & $*$ & $*$ & $*$ & $*$ & $* *$ & $*$ & $*$ & & 8 \\
\hline $\begin{array}{l}\text { Weghofer } \\
\text { et al., } 2008\end{array}$ & * & $*$ & $*$ & $*$ & $* *$ & $*$ & $*$ & $*$ & 9 \\
\hline $\begin{array}{l}\text { Verpoest } \\
\text { et al., } 2008\end{array}$ & $*$ & & $*$ & $*$ & & $*$ & $*$ & & 5 \\
\hline $\begin{array}{l}\text { Haaf } \\
\text { et al., } 2009\end{array}$ & & & $*$ & $*$ & $* *$ & & $*$ & $*$ & 6 \\
\hline $\begin{array}{l}\text { Weghofer } \\
\text { et al., } 2009\end{array}$ & $*$ & $*$ & $*$ & $*$ & $* *$ & $*$ & $*$ & $*$ & 9 \\
\hline $\begin{array}{l}\text { Rubio } \\
\text { et al., } 2010\end{array}$ & $*$ & $*$ & $*$ & $*$ & $* *$ & $*$ & $*$ & & 8 \\
\hline $\begin{array}{l}\text { Kyrou } \\
\text { et al., } 2011\end{array}$ & $*$ & $*$ & $*$ & $*$ & $* *$ & $*$ & $*$ & $*$ & 9 \\
\hline $\begin{array}{l}\text { Massie } \\
\text { et al., } 2011\end{array}$ & $*$ & $*$ & * & $*$ & & $*$ & $*$ & $*$ & 7 \\
\hline $\begin{array}{l}\text { Ata } \\
\text { et al., } 2012\end{array}$ & $*$ & * & $*$ & $*$ & $* *$ & $*$ & $*$ & * & 9 \\
\hline $\begin{array}{l}\text { Labarta } \\
\text { et al., } 2012\end{array}$ & $*$ & $*$ & $*$ & $*$ & $* *$ & $*$ & $*$ & & 8 \\
\hline $\begin{array}{l}\text { Sekhon } \\
\text { et al., } 2017\end{array}$ & $*$ & $*$ & * & $*$ & $* *$ & $*$ & $*$ & * & 9 \\
\hline $\begin{array}{l}\text { Labarta } \\
\text { et al., } 2017\end{array}$ & $*$ & $*$ & $*$ & $*$ & $* *$ & $*$ & $*$ & $*$ & 9 \\
\hline $\begin{array}{l}\text { Barash } \\
\text { et al., } 2017\end{array}$ & $*$ & $*$ & $*$ & $*$ & $* *$ & * & $*$ & $*$ & 9 \\
\hline $\begin{array}{l}\text { Venetis } \\
\text { et al., } 2019\end{array}$ & $*$ & $*$ & $*$ & $*$ & $* *$ & $*$ & $*$ & $*$ & 9 \\
\hline $\begin{array}{l}\text { Thorne } \\
\text { et al., } 2019\end{array}$ & $*$ & * & $*$ & * & $* *$ & $*$ & $*$ & $*$ & 9 \\
\hline
\end{tabular}

* Based on the Newcastle-Ottawa scale. A study can be awarded a maximum of one star for each item within the Selection and Exposure categories. A maximum of two stars can be given for Comparability. Evidence was considered low (0-4), moderate $(5-6)$ or high quality (7-9). 